\title{
Constrained Model-Based Predictive Controller for a High-Frequency Low-Power DC-DC Buck Converter
}

\author{
Ghulam Abbas ${ }^{1}$, Umar Farooq ${ }^{2}$, Jason $\mathrm{Gu}^{3}$ and Muhammad Usman Asad ${ }^{4}$ \\ ${ }^{1,4}$ Department of Electrical Engineering, The University of Lahore, Lahore Pakistan \\ ${ }^{2}$ Department of Electrical Engineering, University of the Punjab, Lahore Pakistan \\ ${ }^{3}$ Department of Electrical and Computer Engineering, Dalhousie University Halifax, N. S. Canada \\ 1'ghulam.abbas@ee.uol.edu.pk, ${ }^{2}$ engr.umarfarooq@yahoo.com, ${ }^{3}$ jason.gu@dal.ca, \\ 4usmanasad01@hotmail.com
}

\begin{abstract}
This paper describes a very detailed and comprehensive description of a model predictive controller (MPC) applied to the buck converter working in Continuous Conduction Mode (CCM) to optimize the performance of the converter. The converter is designed for a fixed switching frequency of $1 \mathrm{MHz}$. The proposed model predictive control technique achieves improved set point tracking with minimal overshoot, offers consistent closed loop performance and has short settling time. Although MPC rejects unmeasured disturbances effectively, but it shows poor load and line regulation. Solution has been suggested in order to robustify the load and line regulation. Further neural network based MPC has also been designed. The impact of the MPC parameters such as prediction and control horizons, performance weights, and constraints on the performance is minutely observed. In order to investigate the effectiveness of the proposed algorithms, MPC is compared with the classical well-known PWM-PID controller. MATLAB/Simulink simulation results validate the control design procedure.
\end{abstract}

Keywords: DC-DC Buck Converter, Model Predictive Controller (MPC), PWM-PID, Continuous Conduction Mode (CCM), Neural Network, Robustification

\section{Introduction}

Synchronous buck converters are being extensively used in regulated switched-mode power supplies (SMPS) targeted for portable applications. However, toggling of the switching converters among a set of linear or nonlinear circuit topologies during an operation, nonlinearity caused by the switching action, chaosity which underlines the complexity, etc. make the power switching converters quite complex and complicated. Due to its switching characteristics, the control problems associated with such converters still pose theoretical and practical problems and challenges to the control community [1]. Linear control techniques do not always guarantee the required static and dynamic performance. The paper proposes the more advanced robust control technique i.e. the constrained model predictive control (MPC) to comply with the challenges.

The acceptable performance for the power switching converters could be achieved using the well-established and most commonly used control techniques like PID and lead-lag [2, 3]. PID parameters normally need to be tuned efficiently to obtain the excellent transient response and robustly ensure the system stability. Depending on tuning guidelines, the tuning process sometimes becomes quite sticky and laborious to achieve a good trade-off between settling time and percent overshoot, particularly under the load variation or input supply voltage perturbation. Model predictive control, although its derivation is more intricate than that of the PID controllers, however is far more intelligent than PID as it incorporates the constraints within the optimization [4].

Although many other nonlinear control methods have been studied, such as fuzzy logic control [5], neural network control [6,7] and sliding mode control (SMC) [8] to resolve the aforementioned problems and challenges, but we will apply a promising and in real sense an

Received: February $22^{\text {nd }} 2013$. Accepted: September $2^{\text {nd }}, 2013$ 
advanced model predictive control (MPC) technique to buck converter to address the issues. Model predictive control (MPC), also known as receding-horizon control (RHC), makes explicit use of the process model and its current measurements to calculate the control signal, at each sampling instant over a finite prediction horizon, by minimizing the cost function.

Unlike the conventional control techniques where the designer tunes, often by trial and error, the controller coefficients and gains to indirectly handle the constraints, the sophisticated RHC control technique optimizes the performance index subject to the various hard and soft constraints to meet the best dynamic performance [9] while manifestly amalgamate the additional control information, like the future predicted outputs and the estimates of future disturbances.

Although MPC suffers from the obvious drawbacks like more complex control algorithm derivation as all the computation has to be carried out at every sampling time, need for an appropriate model of the process, difficulties in its implementation although it is now possible to implement MPC with shorter time steps $[10,11]$ and with the help of FPGA [12] and microcontrollers [13] and its restriction to stable systems [14] but it offers potential advantages including its ability and capability of systematically coping with hard constraints on inputs, outputs and states, its suitability for directly addressing multivariable systems, the ability to systematically handle MIMO control problems subject to constraints, its demonstrability, simplicity, richness, practicality, the on-line solution of an open-loop optimal control problem, need limited knowledge of control, compensation for the measurable disturbances and dead times and its ability to handle non-minimal phase processes.

Historically model predictive control techniques have been extensively used to control constrained multivariable process with complex dynamics [15]. [16] proposes an MPC controller, globally asymptotically stable, that behaves just like a linear controller while optimally dealing with constraints during transients. Distributed model predictive control [17] is suggested to regulate the large scale nonlinear process outputs by considering several distinct sets of manipulated inputs. Although MPC finds its significance for the multi-inputs, multioutputs (MIMO) complex and nonlinear processes, but it can equally be applied to singleinput, single-output (SISO) linear processes which also undergo inequality constraints. On the basis of the discrete-time piecewise affine (PWA) modeling of the power converters where the duty cycle ratio is considered as a constrained manipulated variable, the discrete-time model predictive control (MPC) is familiarized particularly in [18, 19].

Sanders et al. showed the benefits of MPC compared to a standard PID controller [20]. Further the duty ratio is bounded in the interval $[0,1]$ in power converters. From an optimization point of view this is a constrained optimization problem. Hence, it is natural to apply the model predictive control (MPC). In addition, during the last decade, neural networks found their extensive use in various control applications [21, 22, 23]. Nonlinear controllers including MPC can be realized using neural networks which use their universal approximation capabilities of the multilayer perceptron [24]. Several studies ensured the promising results of the application of neural network based nonlinear model predictive control to successful industrial applications $[25,26]$. Thus this paper investigates comprehensively the design and analysis of the model predictive controller.

Rather than following the complex mathematics to describe the MPC algorithm, the paper mainly focuses on the systematic and detailed description of the design of not only the generalized MPC controller but also of the MPC controller designed on the basis of neural networks for the buck converter to achieve the fast dynamic response. The paper minutely studies the effect of the parameters associated with MPC on the closed-loop performance as well. The MPC controller is then compared with the classical PID to confirm its effectiveness. The paper is organized as follows: The small-signal mathematical model of the buck converter in the form of transfer function and state-space is reviewed in Section II. Section III describes the detailed design procedure of the three control techniques: model predictive control, neural network based model predictive control and PID controller. The simulation results obtained on 
the basis of these control techniques are given and compared in section IV. Conclusions are drawn in Section V.

\section{Buck Converter Modeling}

Model predictive control requires a well-defined linear dynamic model of the plant. In this section, we give the bird's eye view of the buck converter model.

A buck converter converts a higher unregulated DC input voltage $V_{i n}$ into a lower regulated output voltage $V_{\text {out }}$ at a required voltage level. The block diagram incorporating a buck converter and a feedback loop is illustrated in Figure 1. The converter consists of a power switching network (transistors $Q_{1}$ and $Q_{2}$ ) and a second order LC low pass filter with parasitics such as inductor DC resistance, $R_{L}$, and capacitor equivalent series resistance, $R_{C}$, denoted explicitly. The capacitor ESR, $R_{C}$, results in introducing a zero frequency to the transfer function [27]. The typical values used for the converter are shown in Table 1.

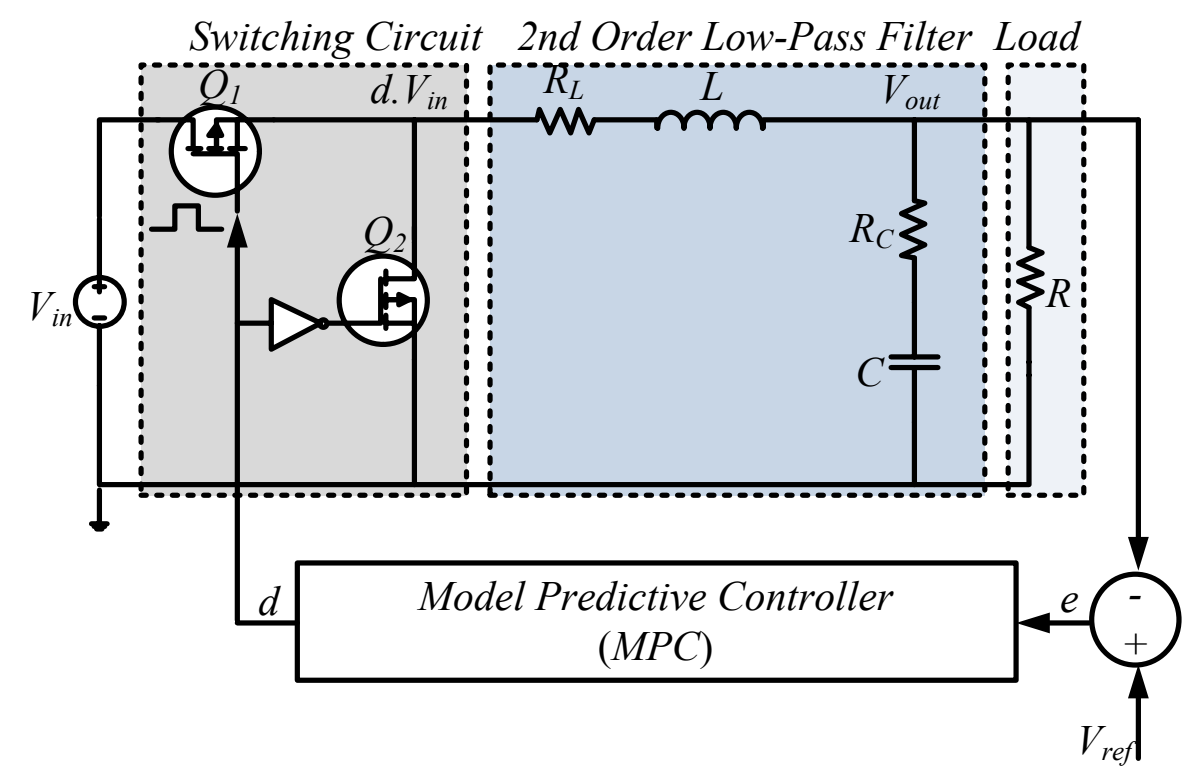

Figure 1. Closed loop analog buck converter system.

Table 1. Buck converter parameters with their typical values

\begin{tabular}{|c|c|c|}
\hline Parameter & Symbol & Value \\
\hline Load Resistance & $R$ & $4.5 \Omega$ \\
\hline Series Resistance of Inductor & $R_{L}$ & $505 \mathrm{~m} \Omega$ \\
\hline Series Resistance of Capacitor & $R_{C}$ & $5 \mathrm{~m} \Omega$ \\
\hline Output Filter Inductor & $L$ & $4.7 \mu \mathrm{H}$ \\
\hline Output Filter Capacitor & $C$ & $4.7 \mu \mathrm{F}$ \\
\hline Input Voltage & $V_{\text {in }}$ & $3.6 \mathrm{~V}$ \\
\hline Output Voltage & $V_{\text {out }}$ & $2 \mathrm{~V}$ \\
\hline Reference Voltage & $V_{\text {ref }}$ & $2 \mathrm{~V}$ \\
\hline Switching Frequency & $f_{s}$ & $1 \mathrm{MHz}$ \\
\hline
\end{tabular}


The open-loop control-to-output voltage transfer function in s-domain is given by [28, 29]:

$$
\begin{aligned}
\frac{v_{\text {out }}(s)}{d} & =\left[\frac{V_{\text {in }}(s)\left(\frac{R}{R+R_{L}}\right)\left(R_{C} C s+1\right)}{L C\left(\frac{R+R_{C}}{R+R_{L}}\right) s^{2}+\left(\frac{L}{R+R_{L}}+C\left(R \square R_{L}\right)+R_{C} C\right) s+1}\right] \\
& =V_{\text {in }}(s)\left(\frac{R}{R+R_{L}}\right)\left(\frac{\frac{s}{\omega_{\text {ZERO }}}+1}{\frac{s^{2}}{\omega_{0}^{2}}+\frac{s}{Q \omega_{0}}+1}\right)
\end{aligned}
$$

with

$$
\omega_{0}=\frac{1}{\sqrt{L C \frac{R+R_{C}}{R+R_{L}}}} ; \omega_{\text {ZERO }}=\frac{1}{R_{C} C} ; Q=\frac{1}{\omega_{0}\left(\frac{L}{R+R_{L}}+C\left(R \square R_{L}\right)+R_{C} C\right)} ;
$$

Where, $\omega_{0}, \omega_{\text {ZERO }}$ and $Q$ represent the LC filter complex double pole frequency, output capacitor ESR zero frequency and the quality factor, respectively.

The current flowing through the inductor $\left(i_{L}\right)$ and the voltage across the capacitor $\left(v_{C}\right)$ are the two state-variables that characterize the buck converter. If we define the state variables as $\mathbf{x}=\left[\begin{array}{ll}i_{L} & v_{C}\end{array}\right]^{T}$, the buck converter dynamics in the form of an LTI state-space can be expressed as:

$$
\begin{array}{r}
\dot{\mathbf{x}}=\mathbf{A x}+\mathbf{B} u \\
y=\mathbf{C x}+\mathbf{D} u
\end{array}
$$

Where, $u$ (duty cycle) is the control input, $x$ is the state vector, and $y$ (the output voltage) is the measurement, and the various matrices are

$$
\begin{array}{ll}
\mathbf{A}=\frac{1}{L C}\left[\begin{array}{cc}
-C \frac{\left(R R_{C}+R R_{L}+R_{C} R_{L}\right)}{R+R_{C}} & -\frac{R C}{R+R_{C}} \\
\frac{R L}{R+R_{C}} & -\frac{L}{R+R_{C}}
\end{array}\right] & \mathbf{B}=\left[\begin{array}{c}
\frac{V_{\text {in }}}{L} \\
0
\end{array}\right] \\
\mathbf{C}=\left[\begin{array}{cc}
\frac{R R_{C}}{R+R_{C}} & \frac{R}{R+R_{C}}
\end{array}\right] & \mathbf{D}=[0]
\end{array}
$$

The continuous system is discretized by using the sampling period $T_{s}=1 \mu \mathrm{s}$ and a zero order hold element. The discrete-time state-space dynamic buck converter system is described by:

$$
\begin{aligned}
\mathbf{x}(k+1) & =\mathbf{\Phi} \mathbf{x}(k)+\boldsymbol{\Gamma} u(k) \\
y(k) & =\mathbf{h x}(k)+\mathbf{J} u(k)
\end{aligned}
$$


Where, $\quad \mathbf{x}(k) \in \mathbf{X} \subseteq \square^{n}, u(k) \in \mathbf{U} \subseteq \square^{m}$ and $y(k) \in Y \subseteq \square^{p}$ represent the system state, control input and output variable, respectively. The matrices $\Phi$ and $\Gamma$ depend on $T_{s}$, the sampling period and are defined as:

$$
\boldsymbol{\Phi}=\mathrm{e}^{\mathrm{AT}_{\mathrm{s}}} ; \quad \boldsymbol{\Gamma}=\left(\int_{0}^{\mathrm{T}_{\mathrm{s}}} \mathrm{e}^{\mathbf{A} \tau} \mathrm{d} \tau\right) \mathbf{B} ; \quad \mathbf{h}=\mathbf{C} ;
$$

With,

$$
\begin{array}{rlrl}
\boldsymbol{\Phi} & =\left[\begin{array}{cc}
0.8766 & -0.1952 \\
0.1952 & 0.9328
\end{array}\right] & \boldsymbol{\Gamma} & =\left[\begin{array}{l}
0.7205 \\
0.0770
\end{array}\right] \\
\mathbf{h} & =\left[\begin{array}{ll}
0.0050 & 0.9989
\end{array}\right] & \mathbf{J}=[0]
\end{array}
$$

This completes the description the buck converter in the form of transfer function and statespace form. The MATLAB toolbox used in the paper may take all forms of the converter.

\section{Controller Design}

In this section, we describe the model predictive control and neural network based model predictive control techniques. Main focus is on MPC while PID is just described for comparison purpose.

\section{A. Model Predictive Controller}

MPC is a control algorithm, based on numerically computing an on-line finite horizon open-loop "optimal" control signal, which solves the optimization problem typically a constrained optimization problem, at each sampling instant, using the current state of the plant as the initial state.

Although different design procedures depending on the presence of constraints may be adopted to describe the MPC algorithm, but we will use the most generalized design procedure as described in [30]. The algorithm calculates a train of the control signal adjustments minimizing a cost function evaluating the difference between the reference signal and the predicted process output in a given future horizon to take the process to the desired regime.

\section{A.1 Basic Control Strategy}

The control strategy applies a receding horizon principle which is schematically presented in Figure 2 for a simplified single-input single-output (SISO) system and is summarized in the following steps:

- At each consecutive sampling instant $k$, a dynamic process model along with models of constraints is applied to predict the future process outputs for an assumed prediction horizon $k \in\left[0, N_{p}\right]$. These predicted outputs $\left\{\hat{y}(k+i \mid k) ; i=0, \ldots, N_{p}-1\right\}$ not only depend on the known past values of control input (manipulated variable) and output signals (controlled variable) up to instant $k$ but also on the future control signals $\left\{u(k+i \mid k) ; i=0, \ldots, N_{m}-1\right\}$ which are applied to the plant. Based on the dynamic plant model, the optimizer generates the future control trajectory as a possible solution, which incorporates the minimization of the objective function while fulfilling the process constraints. The objective function $J$ usually returns the deviation of the predicted output trajectory $\hat{y}(k+i \mid k)$ from the 
reference $r(k+i) . J$ can also include a penalty for the consumption of control strategy.

- At each consecutive sampling instant $k$, a sequence of future control signals $\mathbf{u}(k)=u(k \mid k), u(k+1 \mid k), \ldots, u\left(k+N_{m}-1 \mid k\right) \quad$ is computed, assuming $u(k+h \mid k)=u\left(k+N_{m}-1 \mid k\right) ; h=N_{m}, \ldots, N_{p}$, where $N_{m}$ is the control horizon. The employed notation ' $u(k+h \mid k)$ ' means the prediction of the control input value for the future time $k+h$, performed at the time $k$. The series of control inputs is calculated in such a way that it minimizes the differences between the predicted controlled outputs $\hat{y}(k+i \mid k)$ and the some reference sequence $r(k+i)$ for these outputs over the prediction horizon $N_{p}$. The lower the difference is, the better the setpoint tracking is. Then, only the first element $u(k \mid k)$ of the calculated (control) sequence is applied to control the process, i.e., $u(k)=u(k \mid k)$. The remaining control signals are discarded because at time $k+1$ a new output $y(k+1)$ is not known. At the next sample $(k+1)$, there occurs a new measurement of the process outputs and the controller then obtains new measurements and totally revises its plan. This cycle repeats indefinitely with the prediction horizon of the same length, but shifted by one step forward in time.

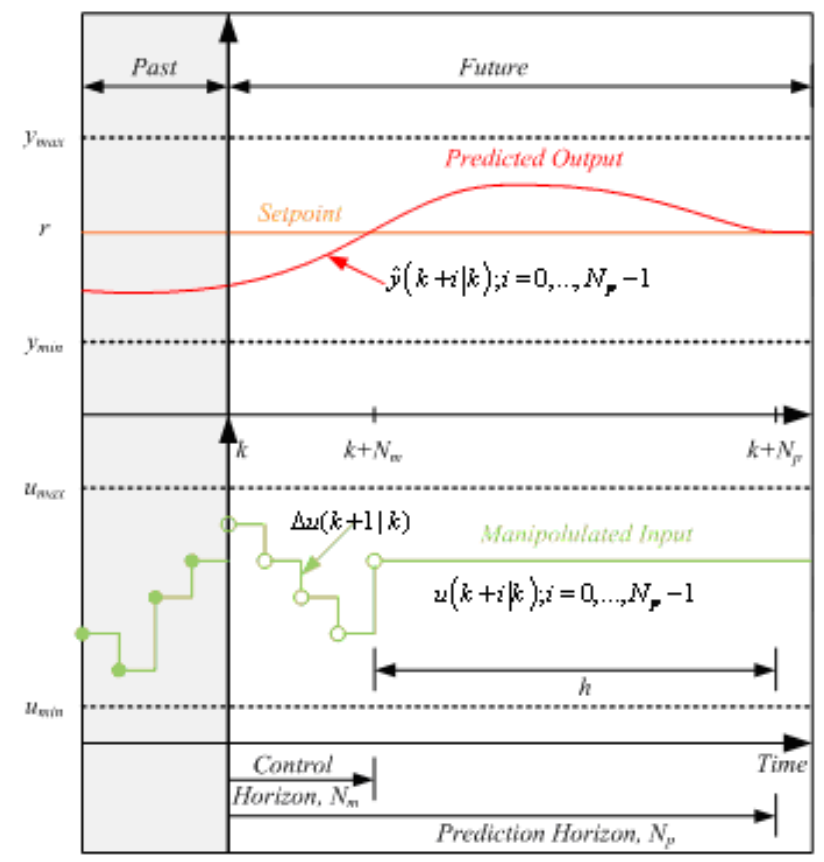

Figure 2. Illustration of working principle of MPC for SISO system.

\section{A.2 Objective Function with Constraints}

For a MIMO system, under the assumption that at each sampling instant $k$, a well-distincted plant dynamic plant model and the estimates of the state variable vector $\mathbf{x}\left(k_{i}\right)$ and the 
measured disturbance vector $\mathbf{x}_{\mathbf{d}}\left(k_{i}\right)$ are available, the standard quadratic objective function (cost function) $J$ needs to be minimized may take the following general form:

$$
\begin{gathered}
J=\left(\begin{array}{l}
\sum_{i=0}^{N_{p}-1} \sum_{j=1}^{n_{y}}\left\{\omega_{i+1, j}^{y} \cdot\left(\hat{y}_{j}(k+i+1 \mid k)-r_{j}(k+i+1)\right)\right\}^{2} \\
+\sum_{i=0}^{N_{m}-1} \sum_{j=1}^{n_{u}}\left\{\omega_{i, j}^{\Delta u} \cdot \Delta u_{j}(k+i \mid k)\right\}^{2} \\
+\sum_{i=0}^{N_{m}-1} \sum_{j=1}^{n_{u}}\left\{\omega_{i, j}^{u} \cdot\left(u_{j}(k+i \mid k)-u_{j \mathrm{target}}(k+i)\right)\right\}^{2}
\end{array}\right)+\rho_{\varepsilon} \varepsilon^{2} \\
\Rightarrow\left\{\Delta \mathbf{u}=\sum_{i=0}^{N_{m}-1} \Delta u(k+i \mid k)\right\}, \varepsilon
\end{gathered}
$$

Where, $N_{p}$ is the prediction horizon and $N_{m}$ is the control horizon. $N_{m}$ is equal to the number of maximal allowed changes of the manipulated variables. $j$ denotes the $j$ th component of a vector and " $(k+i \mid k)$ " denotes the value predicted for time $k+i$ based on the information available at time $k . \hat{y}_{j}$ are the plant controlled or outputs variables (OVs), $u_{j}$ are the plant inputs or manipulated variables (MVs) and $\Delta u_{j}=u_{j}-u_{j-1}$ is the rate of change of the manipulated variable at the $j$ th sampling instant . $n_{u}$ and $n_{y}$ are the number of plant inputs and outputs, respectively. The variables, $\omega_{i+1, j}^{y}, \omega_{i, j}^{u}$ and $\omega_{i, j}^{\Delta u}$ are the non-negative performance weights to penalize the certain components of the variables $y, u$ and $\Delta u$, respectively at certain future time intervals. The smaller the value of $\omega$ the less important is the role of the corresponding term in the cost function. The variable $\varepsilon(\varepsilon \geq 0)$ is the slack variable that is introduced to relax the constraints on $u, \Delta u$, y. The weight $\rho_{\varepsilon}$ on the slack variable $\varepsilon$ penalizes the violation of the constraints. The larger the slack variable weight compared to the manipulated and output variables weights, the more the constraint violation is penalized. The tuning and, therefore, the behavior of the MPC can be adjusted by choosing the appropriate weights $\omega_{i+1, j}^{u}, \omega_{i, j}^{u}$ and $\omega_{i, j}^{\Delta u}$.

Denoting the current sample of a reference for all the measured and unmeasured outputs by $r(k)$, subject to the model equations and to the inequality constraints:

$$
\begin{array}{ll}
\hat{y}_{j}(k+i)=f\left(u_{j}(k), \ldots, u_{j}(k+i-1)\right) & i=0, \ldots, N_{p}-1 \\
y_{j \min }(i)-\varepsilon \leq \hat{y}_{j}(k+i+1 \mid k) \leq y_{j \max }(i)+\varepsilon & i=0, \ldots, N_{p}-1 \\
\Delta u_{j \min }(i) \leq \Delta u_{j}(k+i \mid k) \leq \Delta u_{j \max }(i) & i=0, \ldots, N_{m}-1 \\
u_{j \min }(i) \leq u_{j}(k+i \mid k) \leq u_{j \max }(i) & i=0, \ldots, N_{m}-1 \\
\Delta u(k+h \mid k)=0 & h=N_{m}, \ldots, N_{p}-1 \\
\varepsilon \geq 0 &
\end{array}
$$


Assuming all the input constraints are hard and all the output constraint are soft. In case the reference $r$ is not known in advance, the current reference $r(k)$ is used over the whole prediction horizon, i.e. $r(k+i+1)=r(k)$. The predicted $\hat{y}_{j}(k+i)$ also depends on known and estimated disturbances implicited in (7) as their effects remain constant.

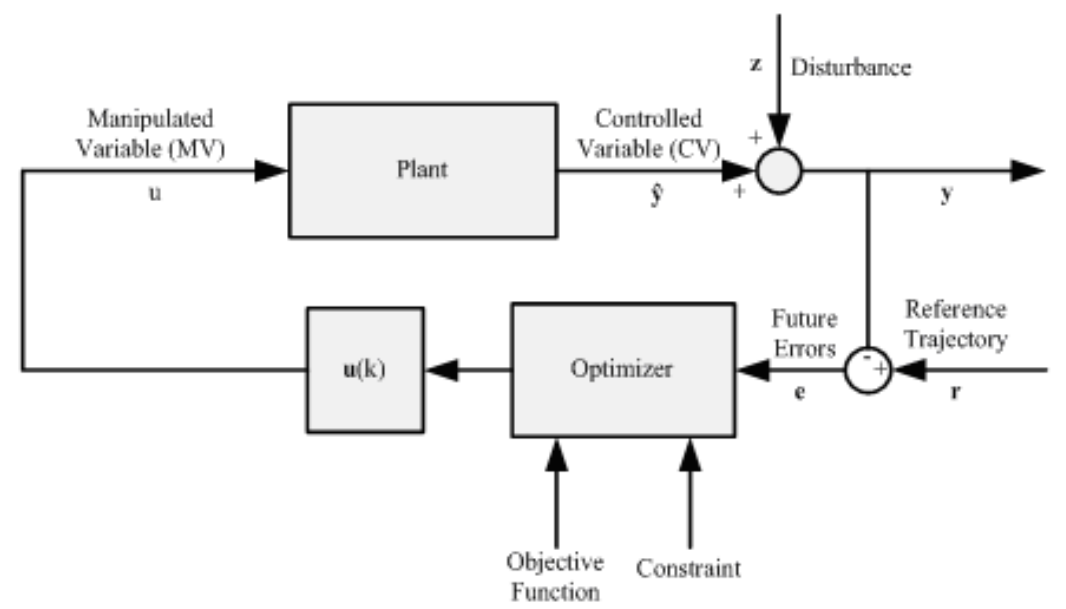

Figure 3. Block diagram representing the basic structure of MPC.

As far as the buck converter system is concerned, it has one manipulated variable i.e. duty cycle ratio $(d)$ and the one output voltage $\left(V_{\text {out }}\right)$ to be controlled. The buck converter is essentially a single-input single output SISO system. The duty ratio, being a control signal, is a sufficiently smooth signal rigidly limited in the closed interval $[0,1]$. The vector $u_{j \text { target }}(k+i)$ which signifies the setpoint for manipulated variable, usually incorporated into the objective function when the number of MVs is greater than that of the OVs, can be excluded from the objective function as we have not imposed any setpoint condition on the duty cycle. The vector $u_{j \text { target }}(k+i)$, in general, is just a sort of lower-priority setpoint.

As the output voltage cannot exceed the input voltage in step-down converter, consequently, we have imposed a constraint on the output voltage i.e. $0 \leq V_{\text {out }} \leq V_{\text {in }}$, unlike the constraint on inductor current described in [31]. In this regard, our approach differs slightly as described in [32]. However this additional soft constraint has negligible effect on the performance. Maximum weight is assigned to the output voltage error in order to achieve the better setpoint tracking.

As can be observed from the cost function that rather than computing the control signal $u$ directly, its gradient, $\Delta u(k \mid k)$ is actually calculated at each step to calculate $u$. Accomplishing this actually allows the control signal to reach the desired level to assist in producing the required output voltage without worsening the performance index $J$. As already remarked that all the remaining samples are rejected and a new optimization problem on the basis of $y(k+1)$ is constructed for the next sampling step $k+1$.

Since the main objective of the MPC is to obtain the fast setpoint tracking, high weight factor is assigned to the error term in the cost function. The control signal variation term usually bears a low weight factor value. In addition, as we have not applied any degree of softness on any of the constraints, the slack variable $\varepsilon$ may be dropped so does its weight. We explicitly have used the hard and soft constraints.

To ensure a steady-state operation under a constant non-zero duty cycle, the difference between two consecutive duty cycles, in the differential form, is computed as: 


$$
\Delta d(k)=d(k)-d(k-1)
$$

The objective function needs to be minimized in case of buck converter system (SISO) thus reduces to:

$$
J\left(N_{p}, N_{m}, \omega_{i+1}^{y}, \omega_{i}^{\Delta u}\right)=\left(\begin{array}{l}
\sum_{i=0}^{N_{p}-1}\left\{\omega_{i+1}^{y} \cdot(\hat{y}(k+i+1 \mid k)-r(k+i+1))\right\}^{2} \\
+\sum_{i=0}^{N_{m}-1}\left\{\omega_{i}^{\Delta u} \cdot \Delta u(k+i \mid k)\right\}^{2}
\end{array}\right)
$$

The constraints on the control input and its gradient and the output signal may be described by the following inequalities:

$$
\begin{array}{ll}
y_{j \min }(i) \leq y_{j}(k+i+1 \mid k) \leq y_{j \max }(i) & i=0, \ldots, N_{p}-1 \\
\Delta u_{j \min }(i) \leq \Delta u_{j}(k+i \mid k) \leq \Delta u_{j \max }(i) & i=0, \ldots, N_{m}-1 \\
u_{j \min }(i) \leq u_{j}(k+i \mid k) \leq u_{j \max }(i) & i=0, \ldots, N_{m}-1
\end{array}
$$

The above ISE (Integral of Squared Error) problem can be converted into an IAE (Integral of Absolute Error) problem by replacing the squared terms with absolute values which place less emphasis on the large deviations.

As can be seen from the optimization problem that the basic tuning parameters of the algorithm are the prediction and control horizons and the non-negative performance weights in the cost function. Some notions regarding the performance weights have already been highlighted.

\section{A.3 Prediction and Control Horizon Selection}

Regarding the choice of $N_{p}$ and $N_{m}$, there are no specific rules. Insensitivity of controller's performance to small perturbations in horizons gives the notions of the $N_{p}$ and $N_{m}$ values. The selection of prediction horizon $N_{p}$ is highly dependent on the sampling period $T_{s}$. In order to ensure the guarantee of the stability, the prediction horizon should be chosen in such a way that it must incorporate the steady-state effect of all the previous control moves. In other words, numerically, it must be equal to the plant's open-loop settling time which is approximately, by rule of thumb, $20-30$ times the sampling period. The prediction horizon is usually taken equal to the plant's open-loop settling time. Relatively small value of control horizon as compared to prediction horizon is selected. The optimization problem, if gives imprecise control predictions, should be solved for other values of $N_{p}$ and $N_{m}$ in order to obtain required performance. An approach has been proposed in [33] to calculate the control input for optimized performance even using the lower value of prediction horizon.

The parameters used by the model predictive controller for the nominal performance (to be shown in simulations results section) are summarized in Table 2.

Table 2. Predictive control parameters

\begin{tabular}{|c|c|c|c|}
\hline Parameter & Value & Parameter & Value \\
\hline Prediction Horizon $\left(N_{p}\right)$ & 14 & Tunning Weight & 0.8 \\
\hline Control Horizon $\left(N_{m}\right)$ & 4 & Control Interval $\left(T_{s}\right)$ & $1 \mu \mathrm{s}$ \\
\hline
\end{tabular}

\section{A.4 Robustification}

The simulation results reveal that MPC shows poor load and line regulation (see the simulation results section), means that when there is a change in load current or input voltage, 
the output voltage does not settle down to its steady-state value, showing some steady-state error. MPC also suffers from the problem in the same way while dealing with the physical limitations, model imperfections, parameter mismatch and unmeasured disturbances. Some methods suggesting the addition of some sort of robustness to MPC can be found in [34, 35]. [36] employs Kalman filter to adjust the output voltage reference against the unmeasured changes in the load resistor. However, we use the simple but effective method to deal with the problem.

The shortcoming arises due to the fact that the control signal applied to the process depends only on the current state, and not on the past history of the process. Consequently the MPC does not take into account the changes in plant dynamics occurring due to the perturbation in supply voltage or load current, thus creating output voltage convergence problem. The controller thus does not provide any guarantee of steady-state zero offset against the load and line transients.

The proposed MPC algorithm can be robustified by introducing an external loop with PI compensator as shown in Figure 4. With the addition of an external loop, the predicted output voltage error which is not available due to the offline realization of the optimization is processed by the PI compensator before the MPC action to move the reference $V_{r e f}$.

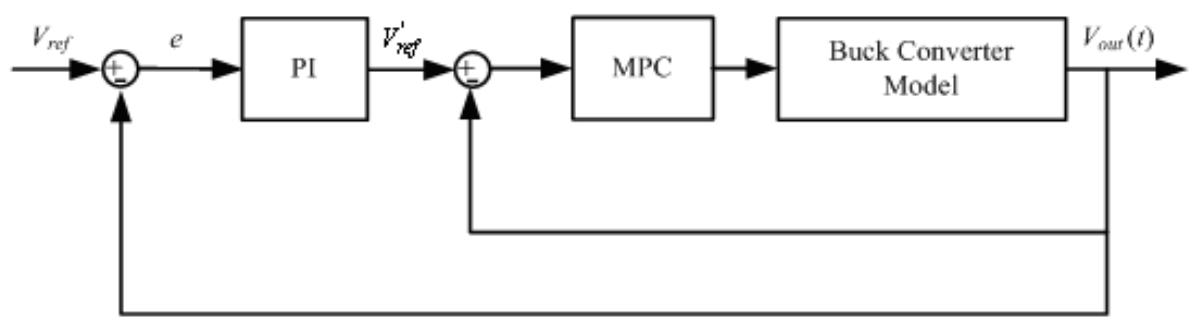

Figure 4. Double-loop control system to ensure steady-state error free tracking.

The newly resulted reference signal $V_{r e f}^{\prime}$ to MPC is expressed as:

$$
V_{r e f}^{\prime}=K_{p} e(t)+K_{i} \int_{0}^{t} e(t) d t \text { where } e(t)=\left(V_{\text {ref }}-V_{\text {out }}\right)
$$

Surely now $\left(V_{\text {ref }}^{\prime}-V_{\text {out }}\right)$ is the manipulated variable to MPC under the new configuration. It has been learned that MPC supported by PI controller ensures offset-free tracking. The PI controller in the external loop of the system adjusts the output voltage reference of MPC in such a way that it safeguards the output voltage tracking under the load and line variation.

We have used the same integral term of the PID compensator (to be discussed later). The proportional constant is found by a hit and trial method. The integrator assists in eliminating the steady-state tracking error whereas the proportional constant tends to ameliorate the transient response without effectively disturbing the system dynamics. The PI compensator employed in the external loop is:

$$
G_{P I}(s)=K_{p}+\frac{K_{i}}{s}=0.2+\frac{101336.45}{s}
$$

\section{B. NN Model Predictive Controller [7]}

Neural networks based model predictive controller can be realized by employing Neural Network Toolbox [37] which uses a neural network model of a nonlinear plant. The future plant performance can be predicted using the Network. The controller determines the control input that assists in optimizing the plant performance over a specified future time horizon. 
There involves two steps, namely system identification and predictive control when using neural networks for control of a system.

- The first step involves the training of a neural network by model predictive control in order to perform the system identification. Figure 5 depicts that the prediction error between the plant output and the neural network plant output constitutes the neural network training signal.

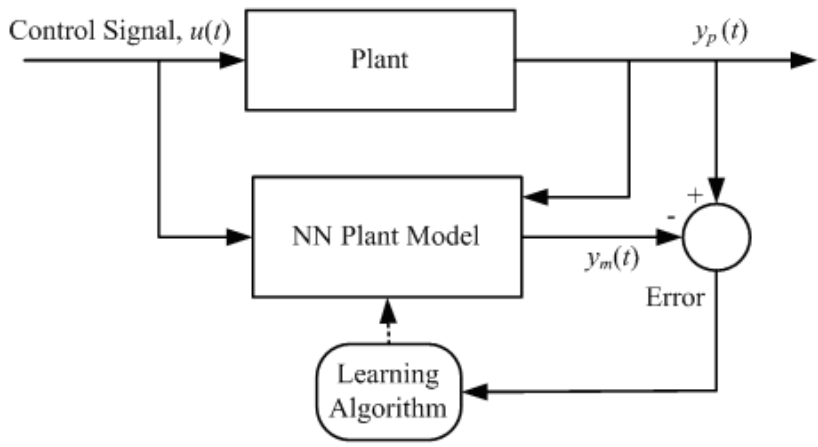

Figure 5. Complete closed-loop control system.

In order to predict the future values of the plant output, $\mathrm{NN}$ plant model uses previous inputs and previous plant outputs. In Figure 6, the inputs to the neural network, namely $y_{p}(t)$ and $u(t)$ denote the current plant output and the control signal to the plant respectively whereas $y_{m}(t+1)$ denotes the predicted plant output.

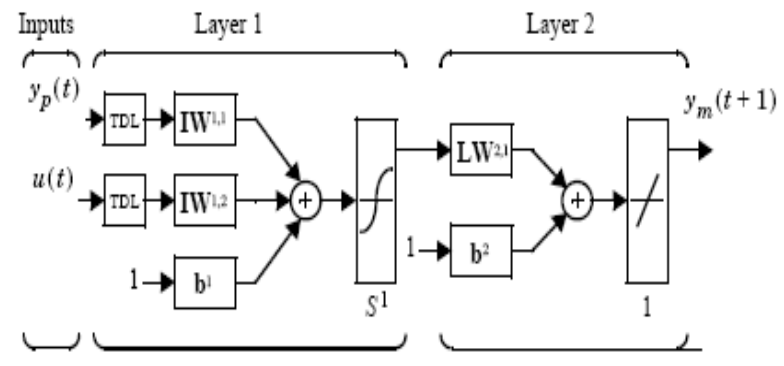

Figure 6. NN plant model [37] (Only two hidden layers are shown).

The output of the network, being the duty cycle, is bounded to values between 0 and 1 . This forces the output layer to use a sigmoid transfer function which follows the four hidden layers of sigmoid neurons of feedforward networks in our case. An appropriate weight $w$ is assigned to each input. The sum of the weighted inputs and the bias constitutes the input to the transfer function. Various activation functions are tabulated in Table 3.

Table 3. Activation functions

\begin{tabular}{|c|c|c|}
\hline Activation Functions & MATLAB Description & Expression/Definition \\
\hline Linear & purelin & $f(x)=x$ \\
\hline Linear-Sigmoid & logsig & $f(x)=\frac{1}{1+e^{-x}}$ \\
\hline Tan-Sigmoid & tansig & $f(x)=\frac{e^{x}-e^{-x}}{e^{x}+e^{-x}}$ \\
\hline
\end{tabular}


The network is trained offline in batch mode using Levenberg-Marquardt (trainlm) training algorithm which optimizes the network weights and biases to minimize the cost function. The cost function to be minimized for feedforward networks is mean square error (MSE) - the average squared error between the network outputs $y_{m}(t)$ and the target outputs $y_{p}(t)-$ is defined as:

$$
F=M S E=\frac{1}{N} \sum_{i=1}^{N}\left(e_{i}\right)^{2}=\frac{1}{N} \sum_{i=1}^{N}\left(y_{m}(t)-y_{p}(t)\right)^{2}
$$

Any standard numerical optimization algorithm based either on the Jacobian of the network errors with respect to the weights or the gradient of the network performance with respect to the network weights can be employed to minimize the cost function. Backpropagation algorithm is used to calculate the gradient [38].

The second step involves the determination of receding horizon technique [39] based predictive controller which uses the plant model to predict future performance over a specified time horizon. The numerical optimization program uses the predictions to calculate the control signal that minimizes the following cost function:

$$
J=\sum_{j=N_{1}=1}^{N_{2}}\left(y_{r}(t+j)-y_{m}(t+j)\right)^{2}+\rho \sum_{j=1}^{N_{u}}\left(u^{\prime}(t+j-1)-u^{\prime}(t+j-2)\right)^{2}
$$

The optimization block which determines the values of the tentative control signal $u$ ' (input to the plant) that minimizes the cost function $J$ and the neural network plant model form the controller (see Figure 7).

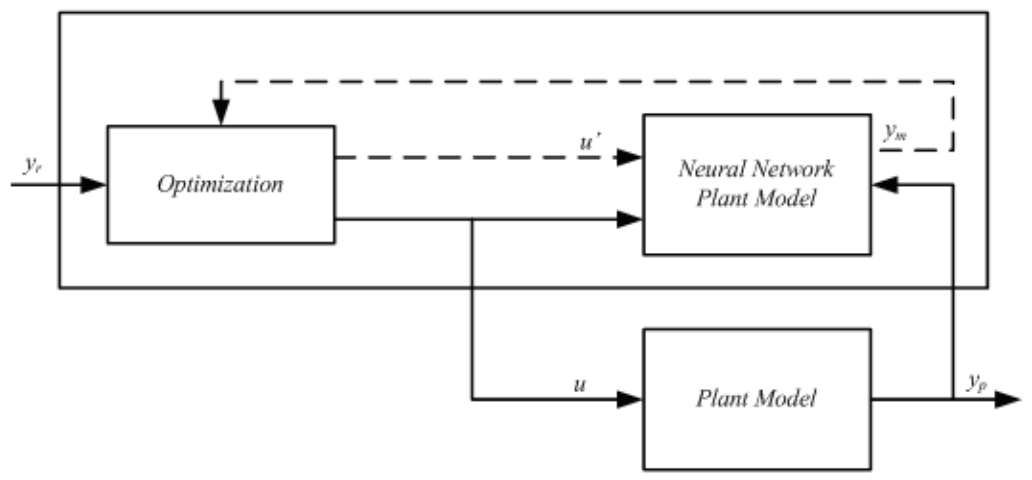

Figure 7. NN plant model with NN model predictive controller [37].

The parameters used by the neural network and predictive control for optimal performance, are listed in Table 4.

Table 4. NN Predictive control parameters

\begin{tabular}{|l|c|l|c|}
\hline \multicolumn{1}{|c|}{ Parameters } & Values & \multicolumn{1}{c|}{ Description } & Value \\
\hline Size of Hidden Layers & 4 & Cost Horizon $\left(N_{2}\right)$ & 14 \\
\hline Training Epochs & 200 & Control Horizon $\left(N_{u}\right)$ & 4 \\
\hline Training Function & 'trainlm' & Control Weighting Factor $(\rho)$ & 0.005 \\
\hline Training Samples & 1000 & Search Parameter $(\alpha)$ & 0.001 \\
\hline
\end{tabular}




\section{PID Controller}

Major concentration has been devoted to MPC controllers. For the sake of comparison, most widely used classical PID compensator with filter is presented in this section.

Assuming the derivative term is made implementable by converting it into a lead term, a continuous-time parallel PID controller, the output of which is the sum of the proportional, integral, and derivative actions, weighted according to the independent gain parameters $K_{p}$, $K_{i}$, and $K_{d}$, is generally given by:

$$
G_{P I D}(s)=K_{p}+\frac{K_{i}}{s}+K_{d}\left(\frac{N s}{s+N}\right)
$$

where $K_{p}, K_{i}$ and $K_{d}$ are the proportional, integral and the derivative constants respectively. The filter pole in the derivative action which lies at $s=-N$ is determined by the filter coefficient $N$. The tuning of the parameters, namely $K_{p}, K_{i}, K_{d}$ and $N$ can be accomplished automatically through SISO Design Tool [40] or Simulink Control Design toolbox. The PID gains using the PID Tuner interface for optimal performance are calculated to be:

$$
K_{p}=1.28, K_{i}=1.01 \times 10^{5}, K_{d}=3.6 \times 10^{-6}, N=1.98 \times 10^{6}
$$

\section{Simulation Results}

In order to investigate the behavior, performance and effectiveness of the constrained MPC described in section III; simulation results using the MATLAB/Simulink environment are presented. Simulation results demonstrate that the proposed MPC offers potential advantages. For this purpose a voltage-mode controlled, synchronous buck converter system which converts $3.6 \mathrm{~V}$ (unless otherwise stated) into $2 \mathrm{~V}$, operating at a switching frequency of $1 \mathrm{MHz}$, is tested and simulated. The buck converter uses the same component values summarized in Table 1. All the measurement noises and the measured and unmeasured disturbances are neglected. The performance of the suggested MPC is also compared with the well-tuned traditional PWM-PID controller.

\section{A. Nominal Results}

Figure 8 exhibits the step response of the buck converter assuming all the initial conditions to be zero. MPC shows an underdamped output voltage response and achieves the performance specifications of maximum overshoot less than $1 \%$ and $7 \mu$ s settling time whereas PWM-PID shows $6 \%$ maximum overshoot and $15 \mu$ s settling time. This shows that MPC, compared to the PWM-PID, takes 2.14 times less time to settle down to its steady-state voltage value of $2 \mathrm{~V}$. NN-MPC almost shows the same performance as that of MPC (settling time $=87 \mu \mathrm{s}$, overshoot $=3.65 \%$ ). The compensated system almost shows the zero steady-state error. The constraint imposed particularly on the duty cycle ratio is efficiently respected. The proposed MPC provides fast closed-loop dynamical performance for the startup without overshoots.

Regarding the NN-MPC, at epoch 200 the validation performance achieves its minimum value of $1.05 \times 10^{-10}$ (see Figure 9). A regression plot shown in Figure 9 clearly ensures the exact linear relationship between the outputs and targets as $R=1$. 
Ghulam Abbas, et al.
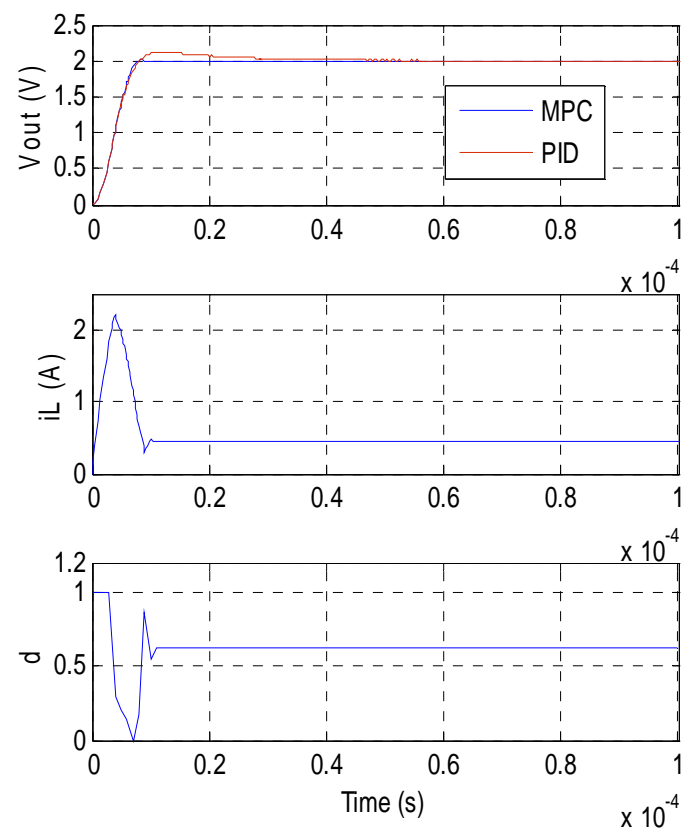

(a)
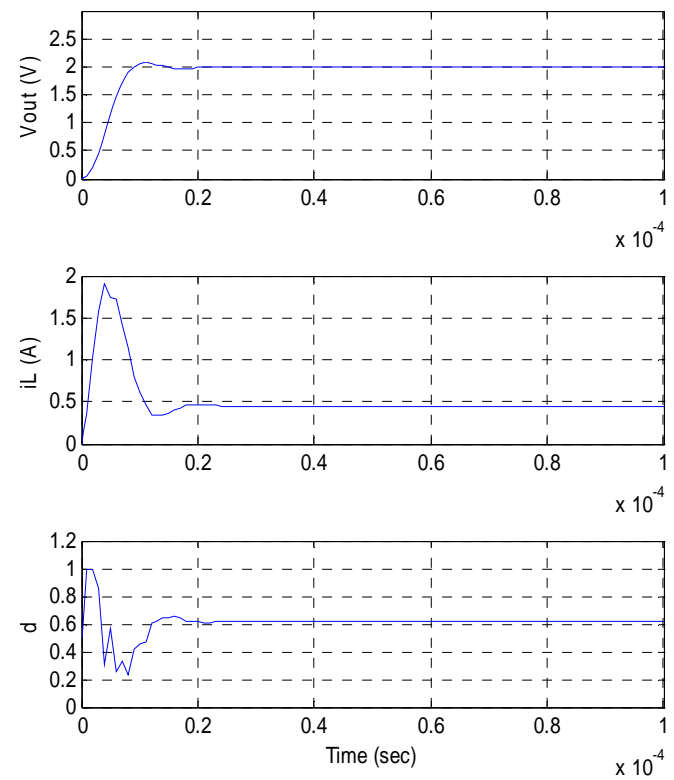

(b)

Figure 8. Nominal performance offered by (a) MPC and (b) NN-MPC. 


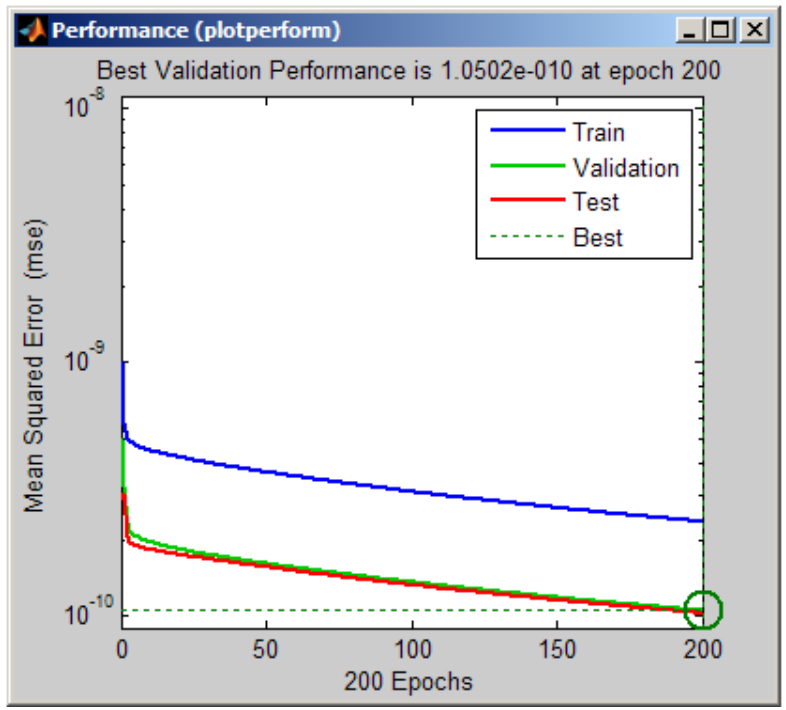

(a)

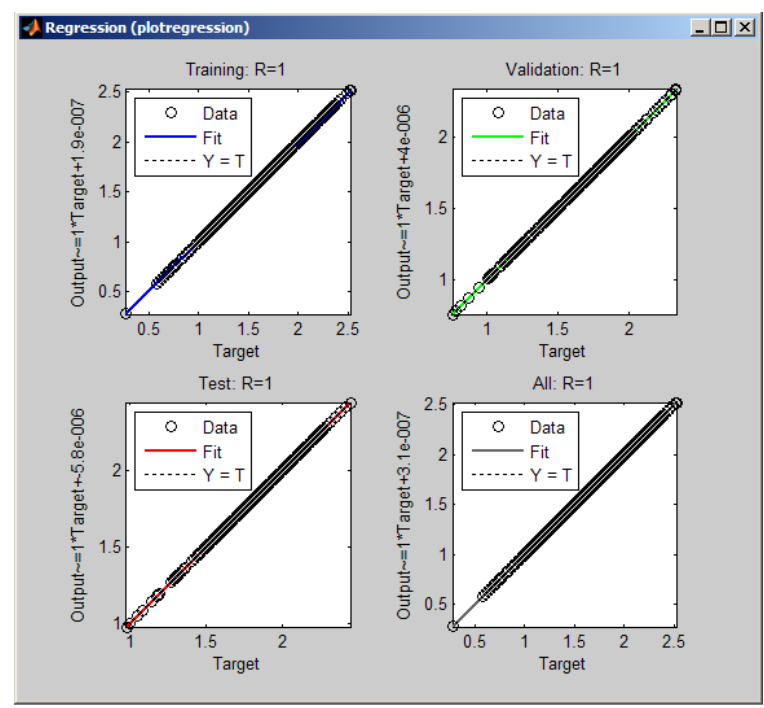

Figure 9. (a) Performance plot. (b) Regression plots.

\section{B. Effect of Prediction and Control Horizons}

The model predictive control strategy is evaluated and investigated using various predictive horizon values. Nominal closed-loop performance improves as $N_{p}$ increases, provided that $N_{m}$ is sufficiently large. Alternatively, if the $N_{p}$ is large enough, increase in $N_{m}$ may improve the performance. However $N_{m}$ is usually kept lower than $N_{p}$. Simulation results depicted in Figure 10 remark that better response is achieved for the $N_{p}$ value of 14 and $N_{m}$ value of 4 . The performance roughly remains the same even if we increase the $N_{p}$ value beyond 14, keeping the $N_{m}$ equal to 4 . No significant improvement is observed even for higher values of $N_{p}$ due to the process-model mismatch. We will use $N_{p}=14$ and $N_{m}=4$ as the most efficient values for the other simulation results in order to reduce the computational time as the algorithm calculates the control signal at each sampling instant. MPC with horizon value of 14 results in nearoptimal control. 

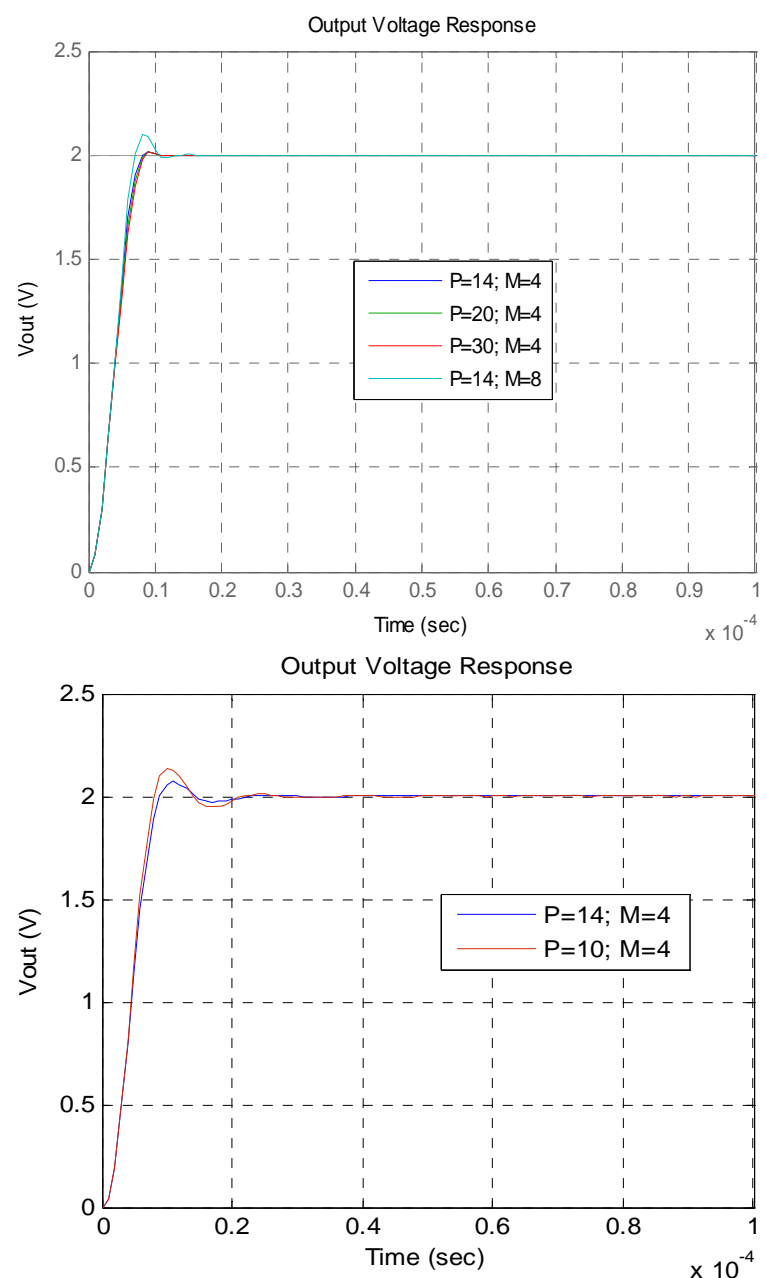

Figure 10. Effect of prediction and control horizons by (left) MPC (right) NN-MPC.

\section{Effect of Weight Tuning}

As mentioned earlier, weighting is one of the tuning parameters to optimize the performance. Well-adjusted tuning weights based controllers lead to optimal control.

The cost function expression incorporates the non-negative performance weights. This clearly shows that the selection of the weights affects the closed-loop performance. The more the weighting, the less active the changes are. As far as the buck converter under consideration is concerned, we have given full weight to the output voltage error in order to ensure the excellent setpoint tracking. Less weight has been assigned to the constrained manipulated variable, ideally zero whereas gradient of the MV gets less weight. Using the nominal values of the prediction and control horizon, the step response clearly shows that sufficiently-weighted control signal drastically deteriorates the setpoint tracking. This observation needs some simple insight. The performance index previously dominated by the steady-state output tracking error where the optimization places all the emphasis on making tracking errors small as control weighting was relatively small, does not remain the same when we give reasonable weight to the gradient of the control signal. This results in poor setpoint tracking. 

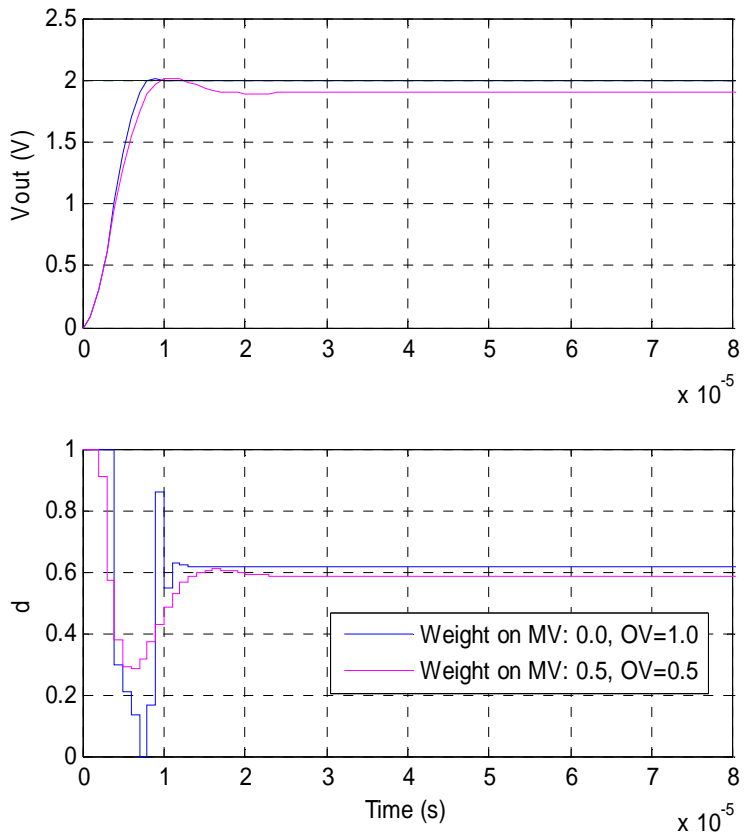

Figure 11. Weight tuning effect shown by MPC.

\section{Effect of Constraints}
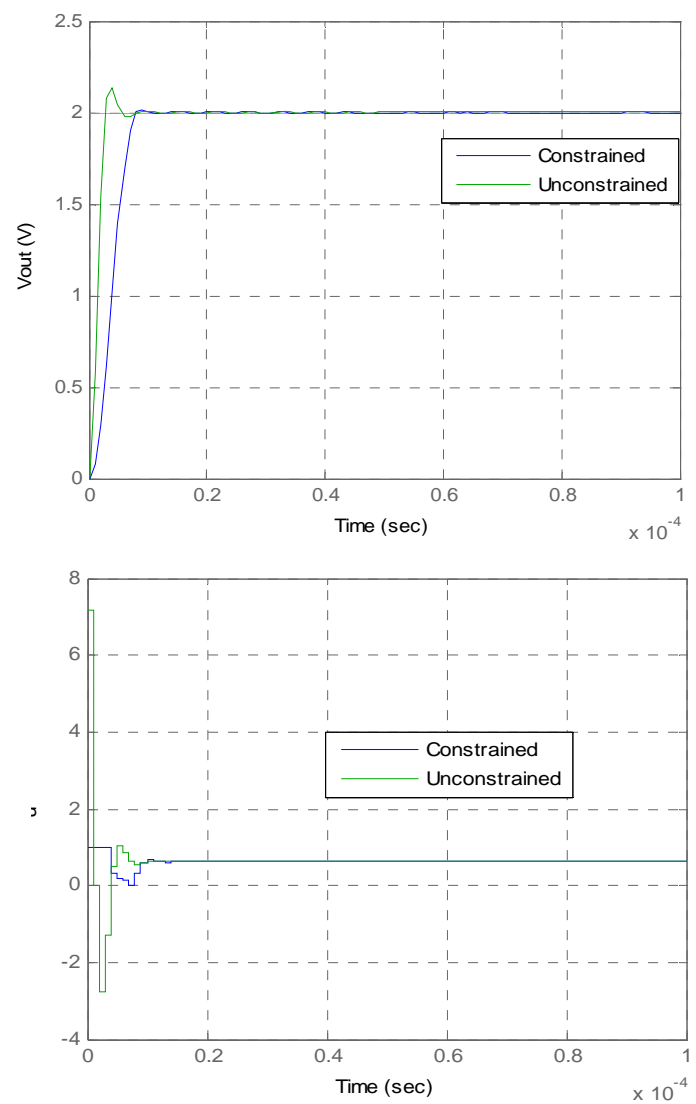

Figure 12. Performance of MPC with and without constraints. 
MPC tends to handle the active constraints, soft and hard, in a systematic way even by ensuring, to some extent, the stability margins and closed-loop performance as that of the unconstrained control law. The control algorithm accomplishes this by incorporating the constraints represented by a set of linear inequalities into the optimization problem described by a cost function. From Figure 12, it is obvious that constrained MPC shows reasonable performance over the whole prediction horizon while meeting constrains imposed on the control as well as on the output signal. However, the unconstrained MPC offers even faster startup response. This is due to the reason that unconstrained control algorithm needs not to calculate the constraints at each sampling time $k$ as does the constrained algorithm. Sometimes, we do not come with well-posed optimization under the given constraints. The constraints may be relaxed gradually, in this case, to find the optimal solution.

\section{E. Set Point Tracking}

The results point out that MPC can be used effectively to keep good setpoint tracking. Figure 13 shows that MPC follows the changes in the reference voltage when changed from 2 $\mathrm{V}$ to $3 \mathrm{~V}$ and then from $3 \mathrm{~V}$ to $2 \mathrm{~V}$ with negligible overshoot in the startup whereas PWM-PID controller shows somewhat increased overshoot in the startup as well as at the time of transients. Thus MPC shows good output voltage tracking characteristics. NN-MPC also demonstrates good tracking of the reference trajectory with low control energy consumption (simulations results are not shown for saving space purposes).

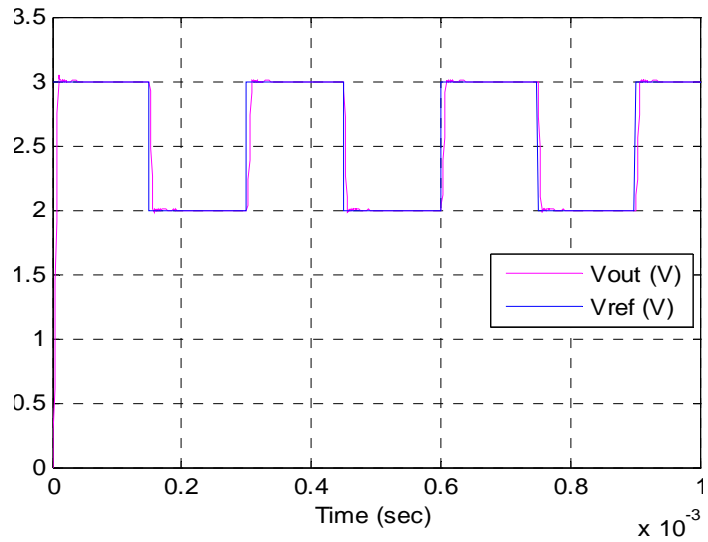

(a)

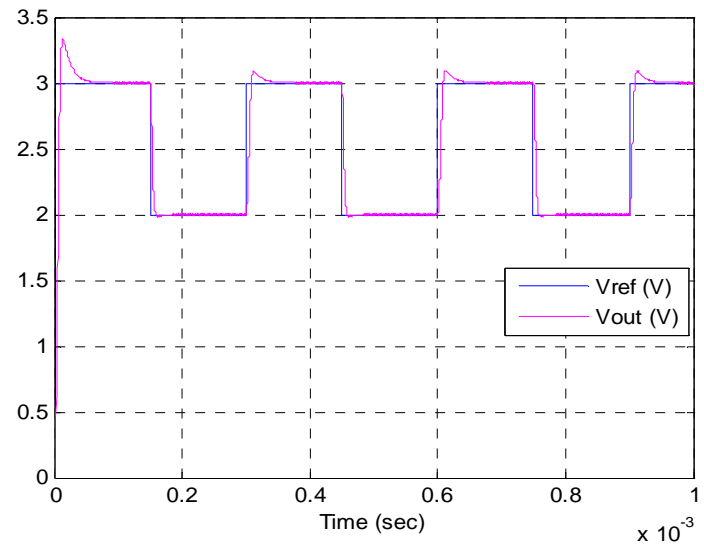

(b)

(c)

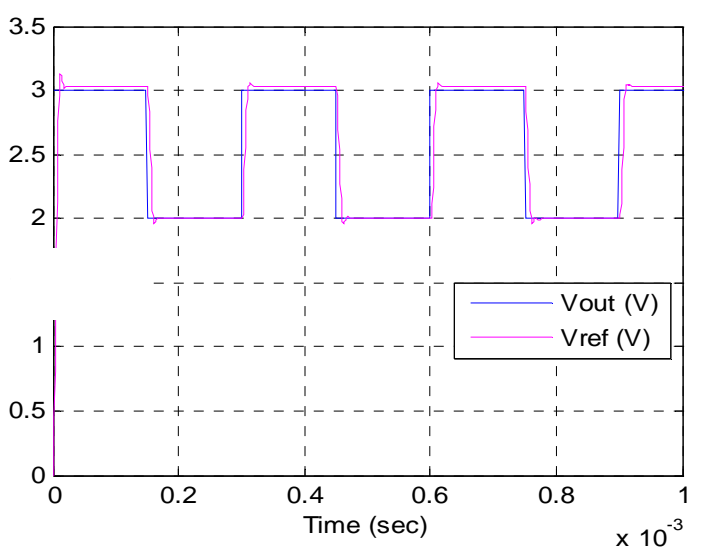

Figure 13. Setpoint tracking offered by (a) MPC, (b) PWM-PID, and (c) NN-MPC 


\section{F. Load Regulation}

The MPC algorithm surely depends on the load resistance. In order to investigate the dynamic response of the system, the load resistance is changed from $4.5 \Omega$ to $9 \Omega$ and then from $9 \Omega$ to $4.5 \Omega(50 \%$ dynamic load change). The simulation results dictate that the output voltage does not converge exactly to the steady-state value, showing the steady state error for the step-up and step-down load changes. However robustification provided to the MPC by adding an external loop ensures the steady-state error free tracking (see Figure 14).
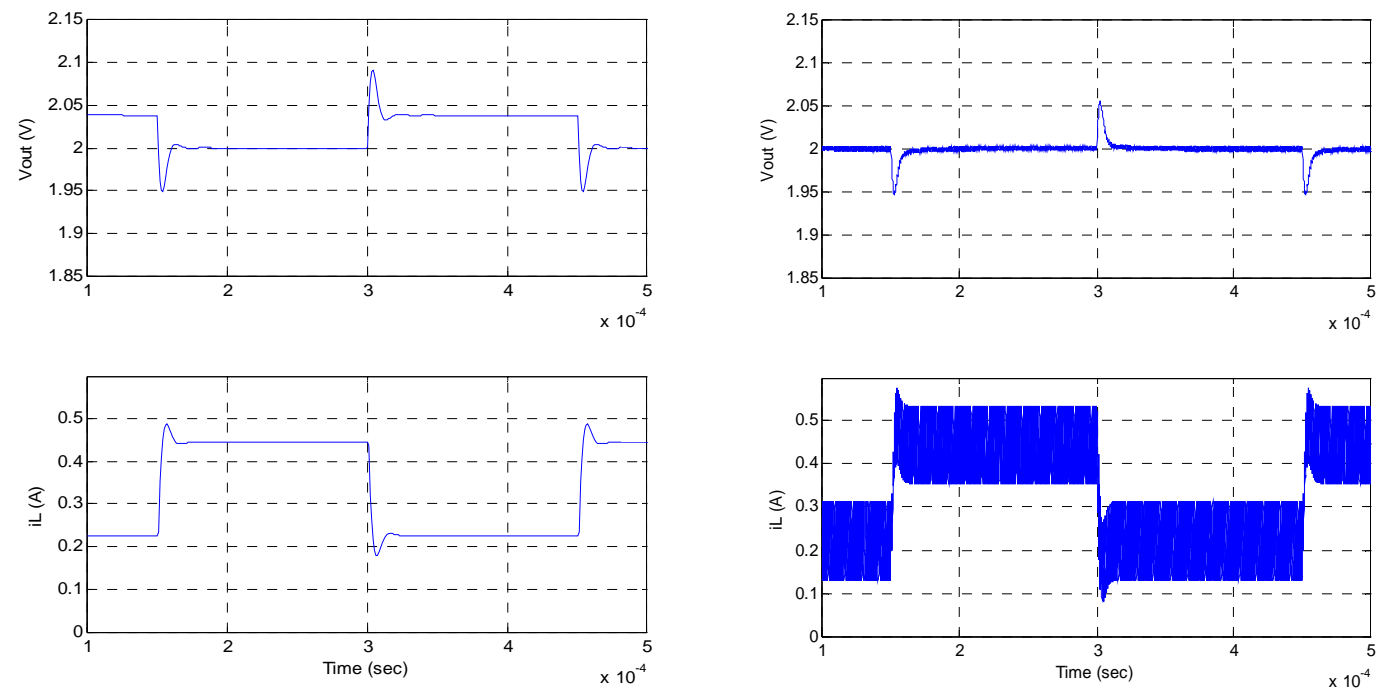

(a)

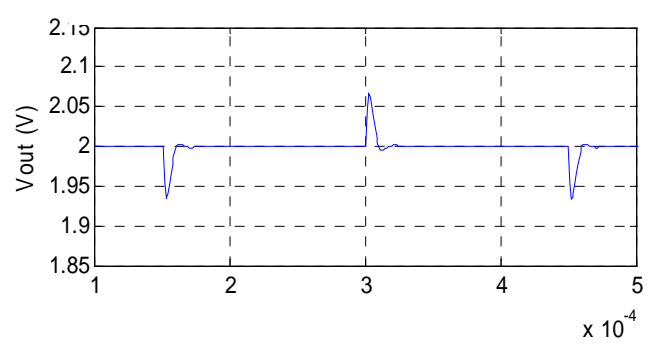

(b)

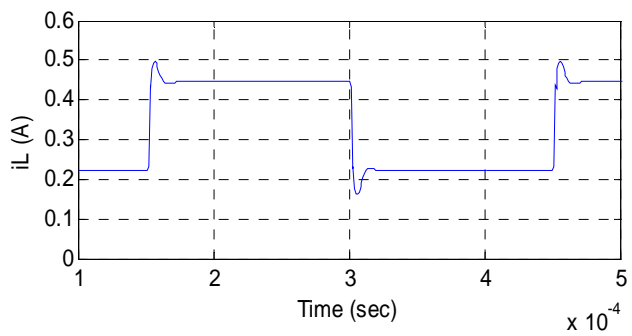

(c)

Figure 14. Load regulation offered by (a) MPC, (b) PWM-PID, and

(c) MPC supported by PI controller.

\section{G. Line Regulation}

The effect of variation in input voltage on the output performance is investigated in Figure 15. It is learned that like load regulation, MPC shows poor line regulation compared to PWMPID controller. The line regulation is evaluated by making a step change in the input voltage to $4.6 \mathrm{~V}$ from its nominal value of $3.6 \mathrm{~V}$ and then from $4.6 \mathrm{~V}$ to $3.6 \mathrm{~V}$ during the steady-state 
operation. However, Figure 15 (c) shows that MPC supported by PI controller shows good line regulation comparable to that of PID.
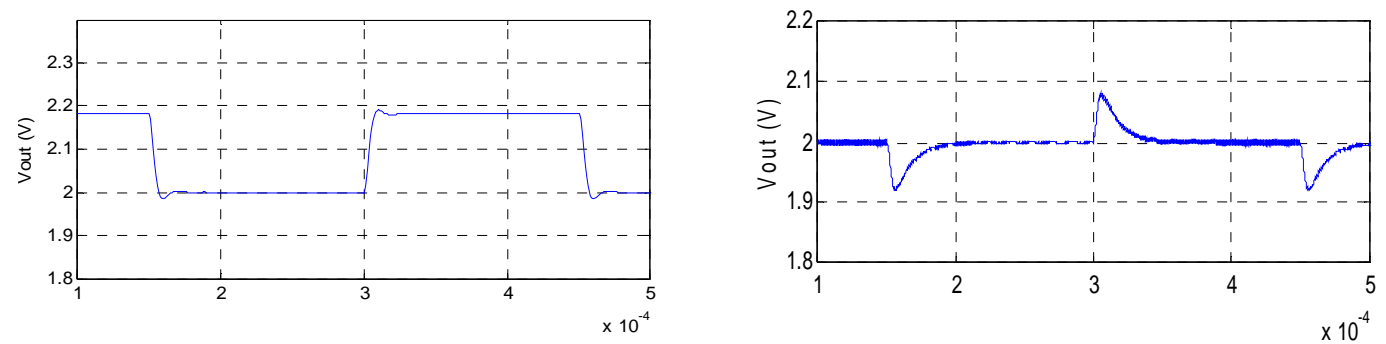

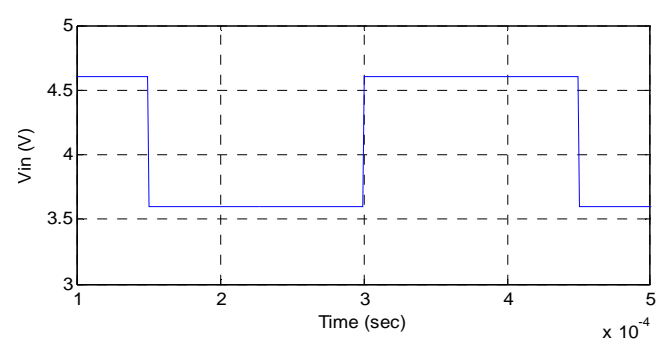

(a)

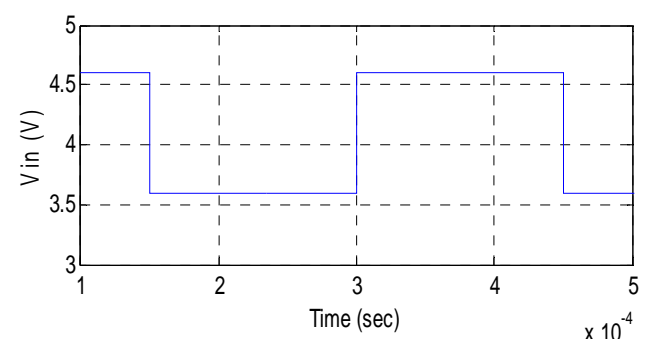

(b)
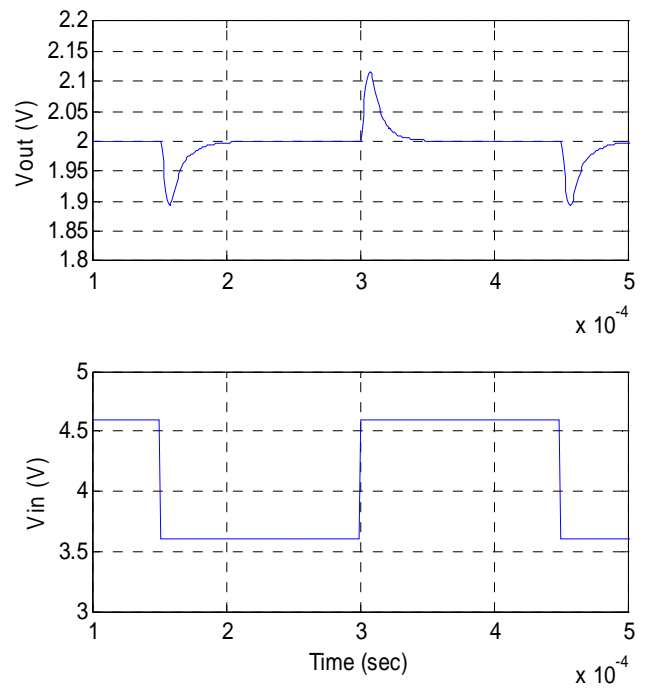

(c)

Figure 15. Line regulation offered by (a) MPC, (b) PWM-PID, and (c) MPC supported by PI controller.

Simulation results highly recommend the model predictive control as an advanced control technique to adequately achieve the excellent static and dynamic performance when applied to single-input, single-output (SISO) buck converter system. 


\section{Conclusions}

In this paper, a model-based predictive control method, based on the general theory and on the neural network model, has been proposed and investigated in a very comprehensive way. The proposed model predictive control scheme successfully minimizes the cost function to achieve better trade-off between fast response and low overshoot for start-up as compared to the PWM-PID. It not only eliminates the maximum overshoot faster but also takes the least time to settle to its steady state value. Effects of constraints, horizons both prediction and control, and performance weights on the closed-loop performance are investigated both theoretically and practically. Excellent load and line regulation have been achieved by introducing an external loop which contains a PI controller.

Compared to the LQR and PWM-PID controllers, MPC handles the constrained problems with complex MIMO structure in an attractive way and shows the well-controlled tuning characteristics. MATLAB/Simulink based simulation results validate the effectiveness of the design.

MPC comes with the disadvantage that the potentially intensive computation has to be performed to calculate the sequence of a control signal at each sampling instant for better setpoint tracking. However the computational issue is no longer relevant as today's computer power is at our disposal.

Future work involves the experimental verification of the simulation results and implementation and testing of the MPC. The performance of the different cost functions can also be investigated for achieving good performance and robustness.

\section{References}

[1] Hebertt J. Sira-Ramirez and Ramón Silva-Ortigoza, "Control Design Techniques in Power Electronics Devices”, 1st Edition, ISBN: 978-1846284588, Springer, 2006.

[2] A. J. Forsyth and S. V. Mollov, "Modeling and Control of DC-DC Converters", Power Engineering Journal, Vol. 12, No. 5, pp. 229-236, 1998.

[3] J-H. Su, J-J. Chen, and D-S. Wu, "Learning Feedback Controller Design of Switching Converters Via MATLAB/Simulink", IEEE Trans. on Education, Vol. 45, No. 4, pp.307-315, 2002.

[4] E. F. Camacho and C. Bordons, "Model Predictive Control", 2nd Edition, SpringerVerlag, 2004.

[5] K. Lian, J. Liou, and C. Huang, "LMI-based integral fuzzy control of DC-DC converters", IEEE Transactions on Fuzzy Systems, 2006, pp. 71-80.

[6] J. Mahdavi, M. R. Nasiri, A. Agah, and A. Emadi, "Application of Neural Networks and State-Space Averaging to DC/DC PWM Converters in Sliding-Mode Operation", IEEE/ASME Trans.on Mechatronics, Vol.10, No.1, pp. 60-67, 2005.

[7] G. Abbas, U. Farooq, and M. U. Asad, "Application of Neural Network Based Model Predictive Controller to Power Switching Converters", International Conference and Workshop on Current Trends in Information Technology (CTIT), pp. 132-136, 26-27 Oct. 2011, Dubai.

[8] J. M. Carraso, J. M. Quero, F. P. Ridao, M. A. Perales, and L.G. Franquelo, "Sliding Mode Control of a DC/DC PWM Converter with PFC Implemented by Neural Network", IEEE Trans. on Circuit Sys. 1: FTA, Vol. 44, No. 8, 1997, pp.743-749.

[9] J. M. Maciejowski, Predictive Control with Constraints. Englewood Cliffs, NJ: PrenticeHall, 2002.

[10] M. Morari and J. H. Lee, "Model predictive control: past, present and future," Comput. Chem. Eng., vol. 23, no. 4/5, pp. 667-682, May 1999.

[11] P. Cortés, M. P. Kazmierkowski, R. M. Kennel, D. E. Quevedo, and J. Rodríguez, "Predictive control in power electronics and drives," IEEE Trans. Ind. Electron., vol. 55, no. 12, pp. 4312-4324, Dec. 2008.

[12] K. V. Ling, S. P. Yue, and J. M. Maciejowski, "An FPGA implementation of model predictive control,” American Control Conference, pp. 1930-1935, Minneapolis, 2006. 
[13] A. K. Abbes, F. Bouani, and M. Ksouri, "A Microcontroller Implementation of Constrained Model Predictive Control", World Academy of Science, Engineering and Technology 80, 2011.

[14] A. Zheng, "Robust Control of Systems Subject to Constraints," Ph.D. Thesis, California Institute of Technonlogy, Pasadena, California, 1995.

[15] A. Rusnák, M. Fikar, K. Najim, and A. Mészáros, "Generalized predictive control based on neural networks," Neural Process, Lett. 4 (1996), pp. 107-112.

[16] S. Di Cairano and A. Bemporad, "Model predictive control tuning by controller matching," IEEE Trans. Automat. Contr., vol. 55, no. 1, pp. 185-190, 2010.

[17] J. Liu, X. Chen, D. Muñoz de la Peña, and P. D. Christofides, "Sequential and iterative architectures for distributed model predictive control of nonlinear process systems," AIChE Journal, pp. 2137-2149, 2010.

[18] T. Geyer, G. Papafotiou, and M. Morari "Model predictive control in power electronics: A hybrid systems approach," Proc. CDC-ECC'05, pp. 5606-5611, 2005.

[19] A. G. Beccuti, G. Papafotiou, M. Morari, S. Almer, H. Fujioka, A. Wernrud, Rantzer, M. Baja, H. Cormerais, and J. Buisson, "Hybrid control techniques for switched-mode DC-DC converters Part II: The step-up topology," Proc. ACC'07, pp. 5464-5471, 2007.

[20] C. F. W. Sanders, M. J. Hounslow, and F. J. Doyle III, "Model Predictive Control of Wet Granulation Using an Experimentally Validated Population Balance Model," AIChE Annual Meeting (2006), San Francisco.

[21] K. S. Narendra and K. Parthasarathy, "Identification and control of dynamical systems using neural networks," IEEE Trans. on Neural Networks, vol. 1, no. 1, pp. 4-27, 1990.

[22] G. W. Ng, "Application of Neural Networks to Adaptive Control of Nonlinear Systems," Research Studies Press Ltd., Taunton, Somerset, England, 1997.

[23] O. Omidvar and D. L. Elliot (Eds.), "Neural Systems for Control," Academic Press, San Diego, 1997.

[24] M. T. Hagan and H. B. Demuth, "Neural Networks for Control", Proceedings of the 1999 American Control Conference, San Diego, CA, 1999, pp. 1642-1656.

[25] A. Muthuramalingam, S. Himavathi, and E. Srinivasan, "Neural Network Implementation Using FPGA: Issues and Application," International Journal of Information and Communication Engineering, vol. 4, no. 2, Winter 2008.

[26] K. O. Temeng, P. D. Schnelle, and T. J. McAvoy, "Model predictive control of an industrial packed bed reactor using neural networks", J. Process Control 5 (1995), pp. 19-27.

[27] Alejandro Oliva and Simon Ang, "Power-Switching Converters," 3rd Edition, Taylor \& Francis Publisher, 2010, ISBN: 143981533X, 9781439815335.

[28] R. D. Middlebrook and S. Cuk, "A General Unified Approach to Modeling SwitchingConverter Power Stages," IEEE Power Electronics Specialists Conference (PESC), 1976, pp. 18-34.

[29] B. Johansson, "DC-DC Converters, Dynamic Model Design and Experimental Verification," Dissertation LUTEDX/(TEIE-1042)/1-194/(2004), Dep. of Industrial Electrical Engineering and Automation, Lund University, Lund, 2004.

[30] A. Bemporad, M. Morari, and N. L. Ricker, "Model Predictive Control Toolbox 3 User's Guide", The MathWorks, Inc. 2005-2010.

[31] A. Bemporad, N. Ricker, and J. Owen, "Model predictive control-New tools for design and evaluation," in American Control Conference, Boston, MA, 2004, pp. 5622-5627.

[32] T. Geyer, G. Papafotiou, and M. Morari, "On the optimal control of switch-mode DCDC converters," in Hybrid Systems: Computation and Control, vol. 2993, pp. 342-356, Lecture Notes in Computer Science. Springer-Verlag, March 2004.

[33] A. Zheng and W. Zhang, "Nonlinear Predictive Control: Theory and Practice," Chapter 8: Computationally Efficient Nonlinear Model Predictive Control Algorithm for Control of Constrained Nonlinear Systems. IEE Control Engineering Series, 2001. 
[34] G. Pannocchia and E. C. Kerrigan, "Offset-free receding horizon control of constrained linear systems," AIChE J., vol. 51, no. 12, pp. 3134-3146, 2005.

[35] G. Pannocchia, "Robust model predictive control with guaranteed setpoint tracking," $J$. Process Control, vol. 14, no. 8, pp. 927-937, Dec. 2004.

[36] T. Geyer, G. Papafotiou, and M. Morari, "Hybrid model predictive control of the stepdown dc-dc converter," Autom. Control Lab., ETH Zurich, Zurich, Switzerland, Tech. Rep. AUT08-01, 2008 [Online]. Available: http://www.control.ee.ethz.ch.

[37] Mark Hudson Beale, Martin T. Hagan, and Howard B. Demuth, "Neural Network Toolbox TM 7 User's Guide," The MathWorks, Inc. 1992-2010.

[38] R. Rojas, "Neural Networks, A Systematic Introduction," Springer-Verlag, New York, 1996.

[39] D. Soloway and P. J. Haley, "Neural Generalized Predictive Control", Proceedings of the 1996 IEEE International Symposium on Intelligent Control, 1996, pp. 277-281.

[40] Natick, MA. "Control System Toolbox 9 : User's Guide", The Math Works, Inc.
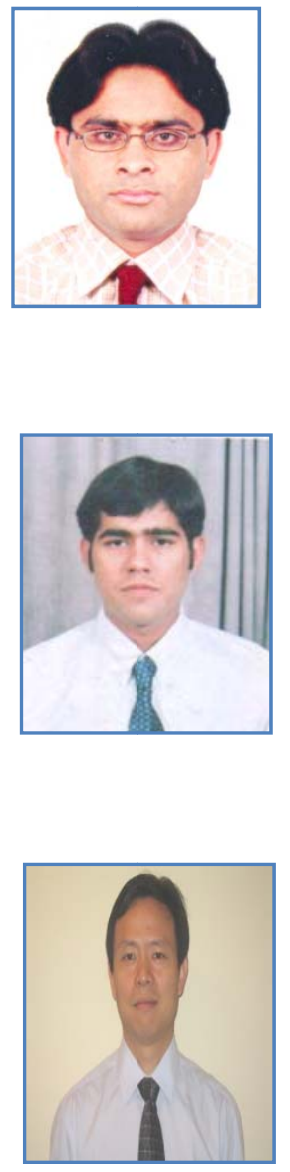

Ghulam Abbas did his B.Sc. in Electrical Engineering from University of Engineering and Technology (UET) Lahore and M.Sc. and Ph.D. both in Electrical Engineering from the Institut National des Sciences Appliquées de Lyon (INSA Lyon) France. He is currently working as an Assistant Professor at the Department of Electrical Engineering, The University of Lahore Pakistan. He has published a number of papers in various IEEE conferences and international journals. His research interests include the modern and intelligent control of power switching converters.

Umar Farooq did his B.Sc. and M.Sc. both in Electrical Engineering from University of Enginering and Technology Lahore, Pakistan in 2004 and 2011 respectively. He has published a number of papers in international conferences and has over eight years of teaching exerience. He is currently working as Assisant Professor at Department of Electrical Engineering, Univesity of The Punjab Lahore. His research interests include intelligent control of nonlinear systems.

Jason Gu received his bachelor's degree from Electrical Engineering and Information Science at the University of Science and Technology of China in 1992, his Master's degree from Biomedical Engineering at Shanghai Jiaotong University in 1995, and $\mathrm{PhD}$ degree from University of Alberta in Canada in 2001. He is currently professor in Electrical and Computer Engineering at Dalhousie University in Canada. He is also a cross-appointed professor in School of Biomedical Engineering and Faculty of Computer Science for his multidisciplinary research work. Dr. Gu's research areas include robotics, biomedical engineering, rehabilitation engineering, neural networks, and control. He has over seventeen years research and teaching experience and has published over 170 conference papers and articles. Twenty one masters, five $\mathrm{PhD}$ and four post doctoral fellows have completed their training under his supervision. He has been the Organization committee for numerous IEEE conferences. He has been the associate editor for following journals: Journal of Control and Intelligent Systems; Transactions on CSME, 
Canada; IEEE Transaction on Mechatronics; International Journal of Robotics and Automation; Unmanned Systems; Journal of Engineering and Emerging Technologies ; IEEE Access.

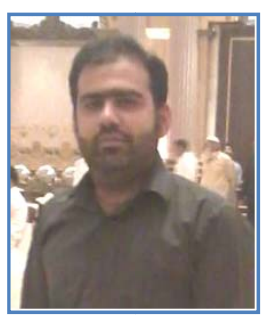

Muhammad Usman Asad did his B.Sc. in Electrical Engineering from University of the Punjab Lahore and is working towards M.Sc. degree in Electrical Engineering from G. C. University Lahore. He was the reciepent of gold and silver medal awards for his papers in $24^{\text {th }}$ and $26^{\text {th }}$ IEEEP International Multi topic Sysmposium held at Bahria University Karachi (2009) and Pakistan Navy Engineering College, National University of Science and Technology (2011) respectively. He is currently working as a Lecturer at Department of Electrical Engineering, The University of Lahore. His research interest include the intelligent control of mobile robots and power systems. 